\title{
Weighted total generalized variation model for Poisson noise removal
}

\author{
Yehu Lv' ${ }^{1}$ \\ (c) Springer Nature Switzerland AG 2019
}

\begin{abstract}
We consider the image denoising problem under Poisson noise. The total generalized variation (TGV)-based method is high efficient in eliminating the staircase effect which often occurs in the total variation-based methods. However, the TGV-based method may produces over-smoothing edges. In order to avoid over-smoothing edges, a weighted total generalized variation (WTGV) model is proposed. Computationally, the WTGV minimization problem is transformed into a saddle-point problem by using the dual technique of optimization. Then, the Chambolle-Pock's first-order primal-dual algorithm is used to solve the transformed saddle-point problem. At last, compared with several existing state-of-theart methods, experimental results demonstrate the performance of our proposed method in edges preservation and staircase effect elimination.
\end{abstract}

Keywords Image denoising - Poisson noise - Weighted total generalized variation (WTGV) · Primal-dual algorithm . Saddle-point problem

\section{Introduction}

The authors in [1-3] mentioned that "in the process of electronic image sampling, transmission and storage, noise is inevitably introduced due to a variety of factors, which affects the further analysis and processing of image". For the Gaussian white noise removal, Rudin et al. [1] presented the Rudin-Osher-Fatemi (ROF) model which can be seen as the total variation (TV)-based method. The ROF model can be written as

$\min _{u} \int_{\Omega}|\nabla u| d x+\frac{\lambda}{2} \int_{\Omega}(u-f)^{2} d x$,

where $\Omega$ is a bounded domain, $u$ is the original image, $f$ is the observed image, $\nabla u$ represents the gradient of $u$, and $\lambda$ is a regularization parameter. As an improvement of the ROF model, Strong et al. [2,3] proposed a weighted TV model which can be written as

$\min _{u} \int_{\Omega} g(x)|\nabla u| d x+\frac{\lambda}{2} \int_{\Omega}(u-f)^{2} d x$, where $g(x)$ represents the weight function. However, according to $[4,5]$, the staircase effect inevitably emerges in the TV-based methods. Recently, Bredies et al. [4] presented the TGV-based method which can eliminate the staircase effect and preserve the edges of image.

In real world, the signal-dependent Poisson noise often occurs in astronomical imaging [6, 7], electronic microscopy $[8-10]$ and medical imaging $[11,12]$ etc. For the Poisson noise removal, Le et al. [13] presented a TV model which can be written as

$\min _{u} \int_{\Omega}|\nabla u| d x+\lambda \int_{\Omega}(u-f \log (u)) d x$.

Le et al. [13] used the gradient descent method to obtain the optimum solution of (3). Because of the convergence rate of the gradient descent method is very slow. Thus, Sawatzky et al. [14] proposed an efficient expectation maximization-total variation (EM-TV) algorithm to accelerate the computational process. In addition, Setzer et al. [15] presented a more efficient alternating split Bregman iterative algorithm to solve (3). Recently, Bonettini et al. [16]

Yehu Lv, mathyehul@163.com | 'Institute of Mathematics, Hebei University of Technology, Tianjin 300401, China. 
used the primal-dual hybrid gradient algorithm to solve the TV-based image denoising problem under Poisson noise. Li et al. [17] proposed the second-order TGV model for Poisson noise removal, and they used the split Bregman iteration algorithm to solve their model.

As is discussed above, we can see that the researchers in [13-17] mainly consider the TV or TGV-based methods without the weight function, and the weighted TV or TGV model is a worthwhile problem. Therefore, combining with the second-order TGV regularization and the weight function $g(x)$, a WTGV model is proposed for Poisson noise removal. Our proposed model can be written as

$\min _{u} \operatorname{WTGV}_{\alpha}^{2}(u)+\tau \int_{\Omega}(u-f \log (u)) d x$,

where $\tau=1 / \lambda$, and the weighted TGV regularization $W T G V_{\alpha}^{2}(u)$ is defined in Sect. 2.

Our main contributions in this paper can be summarized into two aspects listed as follows. First, a WTGV model is presented for Poisson noise removal. Secondly, the WTGV minimization problem is transformed into a saddle-point problem by using the dual technique of optimization. And the high efficient Chambolle-Pock's first-order primal-dual algorithm is used to solve the saddle-point problem.

The outline of this paper is organized as follows. In Sect. 2, the proposed WTGV minimization problem is explained from the view of maximizing a posterior probability. And the WTGV minimization problem is transformed into a saddlepoint problem. In Sect. 3, the Chambolle-Pock's first-order primal-dual algorithm is used to solve the transformed saddle-point problem. In Sect. 4, numerical experiments are given to show that the proposed model can obtain more clearly denoised images. Finally, some conclusions are given in Sect. 5.

\section{The proposed WTGV model for Poisson noise removal}

In this section, the WTGV norm is first transformed into a dual form by introducing two dual variables $p$ and $q$. Then, the WTGV minimization problem is explained from the view of maximizing a posterior probability.

\subsection{The dual form of WTGV norm}

According to [4], the second-order TGV regularization can be formulated as

$T G V_{\alpha}^{2}(u)=\min _{w} \alpha_{1} \int_{\Omega}|\nabla u-w| d x+\alpha_{0} \int_{\Omega}|\varepsilon(w)| d x$,

where $\alpha=\left(\alpha_{0}, \alpha_{1}\right)$ with positive constants $\alpha_{0}$ and $\alpha_{1}, w$ represents an approximation of $\nabla u$, and $\varepsilon(w)$ represents the symmetric gradient. Combining with the definition of the second-order TGV regularization, the proposed WTGV regularization can be formulated as

$W T G V_{\alpha}^{2}(u)=\min _{w} \alpha_{1} \int_{\Omega} g(x)|(\nabla u-w)| d x+\alpha_{0} \int_{\Omega}|\varepsilon(w)| d x$,

where $g(x)$ is the weight function which is as same as the weight function of (2). As showed in [18], the weight function is usually chosen as

$g(x)=\frac{1}{1+c\left|\nabla G_{\sigma} * f\right|^{2}}$,

where $c$ is a positive constant, $*$ is the convolution operation, and

$G_{\sigma}(x)=\frac{1}{2 \pi \sigma^{2}} e^{-\frac{|x|^{2}}{2 \sigma^{2}}}$

is a Gaussian kernel with standard deviation $\sigma$. According to [19], the bounded domain $\Omega$ is replaced by the discretized grid $\Omega_{h}=\{(i, j) \mid 1 \leq i \leq m, 1 \leq j \leq n\}$. And the discretized form of (6) can be written as

$W T G V_{\alpha}^{2}(u)=\min _{w} \alpha_{1} g\|\nabla u-w\|_{1}+\alpha_{0}\|\varepsilon(w)\|_{1}$.

The $l_{1}$-norm is defined as

$\|w\|_{1}=\sum_{i=1}^{m} \sum_{j=1}^{n}=\sum_{i=1}^{m} \sum_{j=1}^{n}\left(\left(w_{1}\right)_{i, j}^{2}+\left(w_{2}\right)_{i, j}^{2}\right)^{1 / 2}$

with $w=\left(w_{1}, w_{2}\right)^{T}$.

According to [20], the conjugate of a function $F(x)$ is defined as $F^{*}(v)=\max _{x}\left(v^{T} x-F(x)\right)$ for any $v$, where $T$ is the matrix transpose operator. If we denote $G_{1}=\nabla u-w$, $G_{2}=\varepsilon(W), F_{1}\left(G_{1}\right)=\alpha_{1} g\left\|G_{1}\right\|_{1}, F_{2}\left(G_{2}\right)=\alpha_{0}\left\|G_{2}\right\|_{1}$. For the function $F_{1}\left(G_{1}\right)$, if we let $v=p$, then its conjugate is

$$
\begin{aligned}
F_{1}^{*}(p) & =\max _{G_{1}} p^{T} G_{1}-F_{1}\left(G_{1}\right)=\max _{G_{1}} p^{T} G_{1}-\alpha_{1} g\left\|G_{1}\right\|_{1} \\
& =\left\{\begin{array}{l}
0, \text { if }|p| \leq \alpha_{1} g, \\
\infty, \text { otherwise. }
\end{array}\right.
\end{aligned}
$$

Similarly, for the function $F_{2}\left(G_{2}\right)$, if we let $v=q$, then its conjugate is

$F_{2}^{*}(q)=\left\{\begin{array}{l}0, \text { if }\|q\|_{\infty} \leq \alpha_{0} \\ \infty, \text { otherwise }\end{array}\right.$

Because of the functions $F_{1}$ and $F_{2}$ are closed and convex, then we have the second conjugates $F_{1}^{* *}=F_{1}$ and $F_{2}^{* *}=F_{2}$. By combining with (11) and (12) as well as the definition of conjugate function, we have 


$$
\begin{aligned}
F_{1}(\nabla u-w) & =\max _{p}(\nabla u-w)^{T} p-F_{1}^{*}(p) \\
& =\max _{p}\langle\nabla u-w, p\rangle-F_{1}^{*}(p)
\end{aligned}
$$

as well as

$$
\begin{aligned}
F_{2}(\varepsilon(w)) & =\max _{q}(\varepsilon(w))^{T} q-F_{2}^{*}(q) \\
& =\max _{q}\langle\varepsilon(w), q\rangle-F_{2}^{*}(q),
\end{aligned}
$$

where the inner product is defined as $\langle u, f\rangle=\sum_{i=1}^{m} \sum_{j=1}^{n} u_{i, j} f_{i, j}$ for $u, f \in \mathbb{R}^{m \times n}$. And we denote

$\delta_{P}(p)=F_{1}^{*}(p)=\left\{\begin{array}{ll}0, & \text { if } p \in P, \\ \infty, & \text { otherwise, }\end{array}\right.$,

$\delta_{Q}(q)=F_{2}^{*}(q)= \begin{cases}0, & \text { if } q \in Q, \\ \infty, & \text { otherwise }\end{cases}$

with

$P=\left\{p=\left(p_{1}, p_{2}\right)^{T}|| p \mid \leq \alpha_{1} g\right\}$,

$Q=\left\{q=\left(\begin{array}{ll}q_{11} & q_{12} \\ q_{21} & q_{22}\end{array}\right) \mid\|q\|_{\infty} \leq \alpha_{0}\right\}$

where

$\left|p_{i, j}\right|=\sqrt{\left(p_{1}\right)_{i, j}^{2}+\left(p_{2}\right)_{i, j^{\prime}}^{2}}$

and $\|q\|_{\infty}=\max _{i, j}\left|q_{i, j}\right|$, with

$\left|q_{i, j}\right|=\sqrt{\left(q_{11}\right)_{i, j}^{2}+\left(q_{12}\right)_{i, j}^{2}+\left(q_{21}\right)_{i, j}^{2}+\left(q_{22}\right)_{i, j}^{2}}$.

By combining with (13), (14) and (15), the minimization problem (9) can be reformulated as the dual form

$\min _{w} \max _{p \in P, q \in Q}\langle\nabla u-w, p\rangle+\langle\varepsilon(w), q\rangle-\delta_{P}(p)-\delta_{Q}(q)$,

where $p$ and $q$ are the dual variables. The gradient operator $\nabla$ is approximated by $\nabla=\left(\partial_{1}^{+}, \partial_{2}^{+}\right)^{T}$, and $\varepsilon(w)$ is approximated by

$\varepsilon(w)=\left(\begin{array}{lr}\partial_{1}^{+} w_{1} & \frac{1}{2}\left(\partial_{2}^{+} w_{1}+\partial_{1}^{+} w_{2}\right) \\ \frac{1}{2}\left(\partial_{1}^{+} w_{2}+\partial_{2}^{+} w_{1}\right) & \partial_{2}^{+} w_{2}\end{array}\right)$

involving the first-order forward difference operators with suitable boundary conditions

$$
\begin{aligned}
& \left(\partial_{1}^{+} u\right)_{i, j}=\left\{\begin{array}{l}
u_{i+1, j}-u_{i, j}, \quad \text { if } 1 \leq i<m, \\
0, \quad \text { if } i=m,
\end{array}\right. \\
& \left(\partial_{2}^{+} u\right)_{i, j}=\left\{\begin{array}{l}
u_{i, j+1}-u_{i, j}, \\
0, \text { if } j=n .
\end{array} \text { if } 1 \leq j<n,\right.
\end{aligned}
$$

If we let $\nabla^{*}=-\operatorname{div}, \varepsilon^{*}=-\operatorname{div}^{h}$, where $\nabla^{*}$ is the adjoint of $\nabla$ and $\varepsilon^{*}$ is the adjoint of $\varepsilon$. Then, the discretized divergence operator div satisfies $\langle\nabla u, p\rangle=\left\langle u, \nabla^{*} p\right\rangle=-\langle u, \operatorname{div}(p)\rangle$, and the discretized divergence operator $\operatorname{div}^{h}$ satisfies $\langle\varepsilon(w), q\rangle=\left\langle w, \varepsilon^{*}(q)\right\rangle=-\left\langle w, \operatorname{div}^{h}(q)\right\rangle$. According to [4, 19], the discretized divergence operator $\operatorname{div}(p)$ is defined as $\operatorname{div}(p)=\partial_{1}^{-} p_{1}+\partial_{2}^{-} p_{2}$, and the discretized divergence operator $\operatorname{div}(q)$ is defined as

$\operatorname{div}^{h}(q)=\left(\begin{array}{l}\partial_{1}^{-} q_{11}+\partial_{2}^{-} q_{12} \\ \partial_{1}^{-} q_{21}+\partial_{2}^{-} q_{22}\end{array}\right)$

involving the first-order backward difference operators with suitable boundary conditions

$\left(\partial_{1}^{-} u\right)_{i, j}=\left\{\begin{array}{lll}u_{i, j}-u_{i-1, j}, & \text { if } \quad 1<i<m, \\ u_{i, j}, & \text { if } i=1, \\ -u_{i-1, j}, & \text { if } \quad i=m,\end{array}\right.$,

$\left(\partial_{2}^{-} u\right)_{i, j}=\left\{\begin{array}{lll}u_{i, j}-u_{i, j-1}, & \text { if } & 1<j<n, \\ u_{i, j}, & \text { if } j=1, \\ -u_{i, j-1}, & \text { if } j=n .\end{array}\right.$

\subsection{The WTGV minimization problem}

Here, the WTGV minimization problem is explained from the view of maximizing a posterior probability $\bar{P}(u \mid f)$ with regard to $u$. In statistics, the Poisson distribution can be characterized by

$\bar{P}_{\mu}(t)=\frac{e^{-\mu} \mu^{t}}{t !}, t \geq 0$,

where $\mu$ is the mean and standard deviation, $\bar{P}$ represents the probability density function. If we let $u \in \mathbb{R}^{m \times n}$ be the original image and $f=$ Poisson $(u)$ be the observed image, where Poisson(.) represents the Poisson function. According to [13], the Bayes's Law can be written as

$\bar{P}(u \mid f)=\frac{\bar{P}(f \mid u) \bar{P}(u)}{\bar{P}(f)}$.

Maximizing the posterior probability $\bar{P}(u \mid f)$ with regard to $u$ is equivalent to

$\max _{u} \bar{P}(u \mid f) \equiv \frac{\bar{P}(f \mid u) \bar{P}(u)}{\bar{P}(f)}$.

It is clear to see that 


$$
\begin{gathered}
\max _{u} \frac{\bar{P}(f \mid u) \bar{P}(u)}{\bar{P}(f)} \Leftrightarrow \min _{u}-\log (\bar{P}(f \mid u) \bar{P}(u)) \\
\Leftrightarrow \min _{u}-\log (\bar{P}(f \mid u))-\log (\bar{P}(u)) .
\end{gathered}
$$

By combining with the likelihood function

$$
\bar{P}(f \mid u)=\prod_{i, j} \bar{P}_{u_{i, j}}\left(f_{i, j}\right)=\prod_{i, j} \frac{\left(u_{i, j}\right)^{f_{i j}} e^{\left(-u_{i, j}\right)}}{\left(f_{i, j}\right) !}
$$

and the prior distribution function

$\bar{P}(u)=e^{-\lambda W T G V_{\alpha}^{2}(u)}$,

we have

$\log (\bar{P}(f \mid u))=\sum_{i=1}^{m} \sum_{j=1}^{n}\left[f_{i, j} \log \left(u_{i, j}\right)-u_{i, j}-\log \left(\left(f_{i, j}\right) !\right)\right]$

as well as

$\log (\bar{P}(u))=-\lambda T G V_{\alpha}^{2}(u)$.

Thus, combining with (27), (30) and (31), we can obtain the WTGV minimization problem

$\min _{u}\langle\mathbf{1}, u\rangle-\langle f, \log (u)\rangle+\lambda W T G V_{\alpha}^{2}(u)$,

where 1 represents a vector of all ones. Combining with (19), the problem (32) can be reformulated as the saddlepoint problem

$$
\begin{gathered}
\min _{u, w} \max _{p \in P, q \in Q} \tau(\langle\mathbf{1}, u\rangle-\langle f, \log (u)\rangle)+\langle\nabla u-w, p\rangle \\
+\langle\varepsilon(w), q\rangle-\delta_{P}(p)-\delta_{Q}(q) .
\end{gathered}
$$

\section{The Chambolle-Pock's first-order primal- dual algorithm}

The primal-dual algorithm [21-26] has been widely used in the field of image processing because of its efficient iterative pattern. Chambolle and Pock [27] proposed a first-order primal-dual algorithm to solve the saddle-point problem

$\min _{x \in X} \max _{y \in Y} \phi(x)+\langle K x, y\rangle-\Psi(y)$, where $\phi(x)$ and $\Psi(y)$ are proper, convex, lower-semicontinuous functions, $X$ and $Y$ are the constrained sets, $\langle$. represents the inner product, and $K$ is a continuous linear operator. The Chambolle-Pock's first-order primal-dual algorithm [27] is similar to the dual method in [28], which used a predictor-corrector scheme for the primal and dual variables. The Chambolle-Pock's first-order primal-dual algorithm for the saddle-point problem (34) can be written as

$$
\begin{aligned}
& y^{k+1}=\arg \max _{y \in Y}\left\langle K \hat{x}^{k}, y\right\rangle-\Psi(y)-\frac{1}{2 t}\left\|y-y^{k}\right\|_{2^{\prime}}^{2}, \\
& x^{k+1}=\arg \min _{x \in X} \phi(x)+\left\langle K x, y^{k+1}\right\rangle+\frac{1}{2 s}\left\|x-x^{k}\right\|_{2^{\prime}}^{2} \\
& \hat{x}^{k+1}=x^{k+1}+\theta\left(x^{k+1}-x^{k}\right),
\end{aligned}
$$

where the parameters $s, t>0$ are step sizes of the primal and dual variables, respectively, and $\theta$ is a fixed parameter.

\subsection{The Chambolle-Pock's first-order primal-dual algorithm for WTGV model}

In order to apply the Chambolle-Pock's first-order primal-dual algorithm to (33), we need to formulate (33) as the form of (34). Denote

$K=\left(\begin{array}{cc}\nabla & -l \\ 0 & \varepsilon\end{array}\right), x=\left(\begin{array}{l}u \\ w\end{array}\right), y=\left(\begin{array}{l}p \\ q\end{array}\right)$,

$\phi(x)=\tau(\langle\mathbf{1}, u\rangle-\langle f, \log (u)\rangle)$, and $\Psi(y)=\delta_{P}(p)+\delta_{Q}(q)$, where $I$ is the identity matrix. We see that (33) completely fits into the framework of (34). Hence, (35) can be used to solve (33). If we let

$$
\begin{aligned}
R(u, w, p, q)= & \tau(\langle\mathbf{1}, u\rangle-\langle f, \log (u)\rangle)+\langle\nabla u-w, p\rangle \\
& +\langle\varepsilon(w), q\rangle-\delta_{p}(p)-\delta_{Q}(q) .
\end{aligned}
$$

Then, (33) can be rewritten as

$\min _{u, w} \max _{p \in P, q \in Q} R(u, w, p, q)$.

The Chambolle-Pock's first-order primal-dual algorithm for (38) can be summarized into Algorithm 1. 


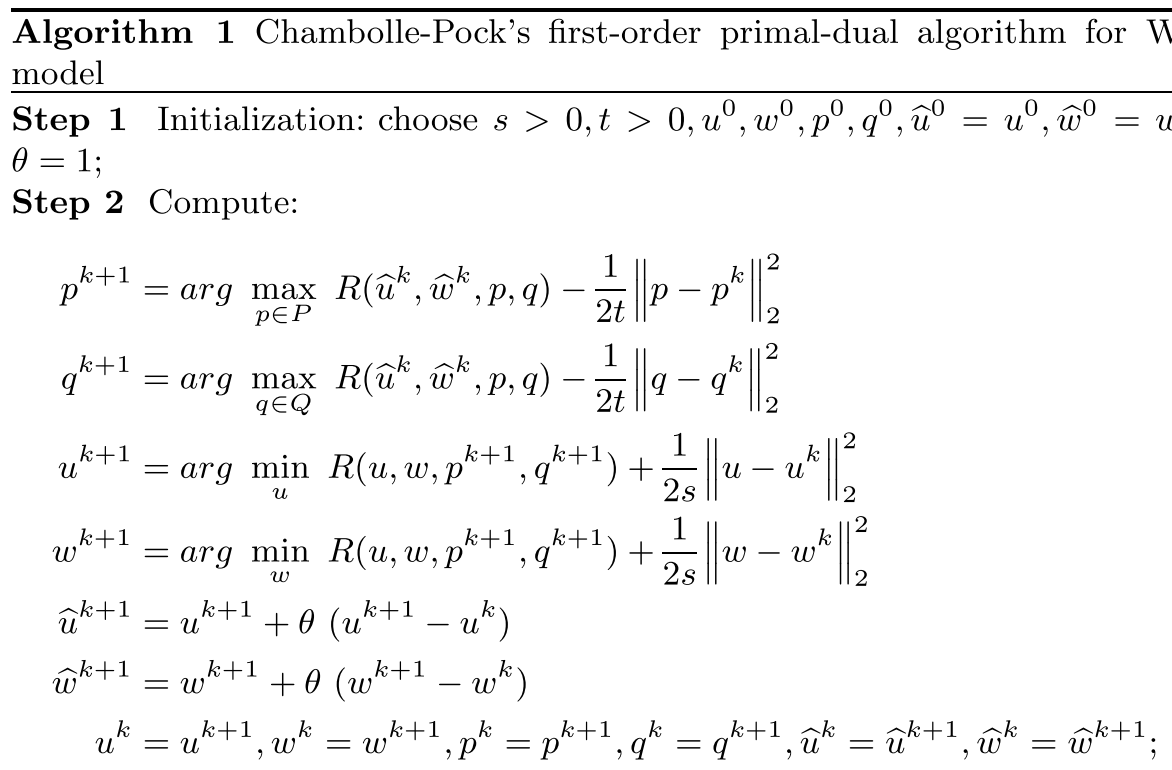

Step 3 When the stopping criterion is fulfilled, the iterative process is stopped; otherwise, let $k=k+1$, then go to Step 2 .

In Algorithm 1, the subproblem for $p$ can be written as

$$
\begin{aligned}
p^{k+1} & =\arg \max _{p \in P}\left\langle\nabla \hat{u}^{k}-\widehat{w}^{k}, p\right\rangle-\delta_{P}(p)-\frac{1}{2 t}\left\|p-p^{k}\right\|_{2}^{2} \\
& =\arg \min _{p \in P} \frac{1}{2 t}\left\|p-p^{k}\right\|_{2}^{2}-\left\langle p, \nabla \hat{u}^{k}-\widehat{w}^{k}\right\rangle \\
& =\arg \min _{p \in P}\left\|p-\left(p^{k}+t\left(\nabla \hat{u}^{k}-\widehat{w}^{k}\right)\right)\right\|_{2}^{2}
\end{aligned}
$$

Combining with the definition of the projection operator $\mathcal{P}_{P}(z)$, i.e., $\mathcal{P}_{P}(z)=\arg \min _{p \in P}\|p-z\|_{2}^{2}$ for any vector $z$, we have

$p^{k+1}=\mathcal{P}_{P}\left(p^{k}+t\left(\nabla \hat{u}^{k}-\widehat{w}^{k}\right)\right)$.

The Euclidean projector $\mathcal{P}_{P}$ represents the projection onto the convex set $P$, which can be computed by

$\mathcal{P}_{p}\left(\bar{p}^{k}\right)=\frac{\bar{p}^{k}}{\max \left(1,\left|\bar{p}^{k}\right| / \alpha_{1} g\right)}$,

where $\bar{p}^{k}=p^{k}+t\left(\nabla \hat{u}^{k}-\widehat{w}^{k}\right)$.

Similarly, for $q$, we have

$q^{k+1}=\mathcal{P}_{Q}\left(q^{k}+t \varepsilon\left(\widehat{w}^{k}\right)\right)$.

If we denote $\bar{q}^{k}=q^{k}+t \varepsilon\left(\widehat{W}^{k}\right)$, then

$q^{k+1}=\frac{\bar{q}^{k}}{\max \left(1,\left|\bar{q}^{k}\right| / \alpha_{0}\right)}$.
The subproblem for $u$ can be written as

$$
\begin{aligned}
u^{k+1}= & \arg \min _{u} \tau(\langle\mathbf{1}, u\rangle-\langle f, \log (u)\rangle) \\
& +\left\langle\nabla u-w, p^{k+1}\right\rangle+\frac{1}{2 s}\left\|u-u^{k}\right\|_{2}^{2} \\
= & \arg \min _{u} \tau(\langle\mathbf{1}, u\rangle-\langle f, \log (u)\rangle) \\
& +\left\langle\nabla u, p^{k+1}\right\rangle+\frac{1}{2 s}\left\|u-u^{k}\right\|_{2}^{2}
\end{aligned}
$$

Its optimality condition is

$u^{2}-u\left(u^{k}+s \operatorname{div}\left(p^{k+1}\right)-s \tau l\right)-s \tau f=0$,

where $I$ is the identity matrix. If we let $\xi^{k}=u^{k}+s \operatorname{div}\left(p^{k+1}\right)$, then the optimality condition can be reformulated as

$u^{2}-u\left(\xi^{k}-s \tau l\right)-s \tau f=0$.

Notice that $u$ should be positive, thus we have

$u^{k+1}=\frac{\left(\xi^{k}-s \tau l\right)+\sqrt{\left(\xi^{k}-s \tau l\right)^{2}+4 s \tau f}}{2}$.

The subproblem for $w$ can be written as 


$$
\begin{aligned}
w^{k+1}= & \arg \min _{w}\left\langle\nabla u-w, p^{k+1}\right\rangle \\
& +\left\langle\varepsilon(w), q^{k+1}\right\rangle+\frac{1}{2 s}\left\|w-w^{k}\right\|_{2}^{2} \\
= & \arg \min _{w}\left\langle-w, p^{k+1}\right\rangle+\left\langle\varepsilon(w), q^{k+1}\right\rangle \\
& +\frac{1}{2 s}\left\|w-w^{k}\right\|_{2}^{2} .
\end{aligned}
$$

Its optimality condition is

$$
-s p^{k+1}-s \operatorname{div}^{h}\left(q^{k+1}\right)+w-w^{k}=0 .
$$

Thus, we have

$w^{k+1}=w^{k}+s\left(p^{k+1}+\operatorname{div}^{h}\left(q^{k+1}\right)\right)$.

\subsection{Convergence analysis}

According to [27], the Chambolle-Pock's first-order primal-dual algorithm enjoys convergence with rate $O(1 / k)$ when $\theta=1$ and the step sizes satisfy $s t\|K\|_{2}^{2}<1$. Thus, an estimate of $\|K\|_{2}^{2}$ is needed. According to the definition of the divergence operator div, we have

Fig. 1 a The original Barbara image, $\mathbf{b}$ the original cameraman image, $\mathbf{c}$ the original satellite image, $\mathbf{d}$ the Barbara image under Poisson noise, $\mathbf{e}$ the cameraman image under Poisson noise, $\mathbf{f}$ the satellite image under Poisson noise

$$
\begin{aligned}
& \|\operatorname{div}(p)\|_{2}^{2}=\sum_{i, j}\left(\left(p_{1}\right)_{i, j}-\left(p_{1}\right)_{i-1, j}+\left(p_{2}\right)_{i, j}-\left(p_{2}\right)_{i, j-1}\right)^{2} \\
& \quad \leq 2 \sum_{i, j}\left[\left(\left(p_{1}\right)_{i, j}-\left(p_{1}\right)_{i-1, j}\right)^{2}+\left(\left(p_{2}\right)_{i, j}-\left(p_{2}\right)_{i, j-1}\right)^{2}\right] \\
& \quad \leq 4 \sum_{i, j}\left[\left(\left(p_{1}\right)_{i, j}\right)^{2}+\left(\left(p_{1}\right)_{i-1, j}\right)^{2}+\left(\left(p_{2}\right)_{i, j}\right)^{2}+\left(\left(p_{2}\right)_{i, j-1}\right)^{2}\right] \\
& \quad \leq 8\|p\|_{2}^{2} .
\end{aligned}
$$

Then, we can obtain $\|\nabla\|_{2}^{2}=\|\operatorname{div}\|_{2}^{2} \leq 8$. Similarly, we can obtain $\|\varepsilon\|_{2}^{2}=\left\|d i v^{h}\right\|_{2}^{2} \leq 8$. After some computations, we obtain the estimate $\|K\|_{2}^{2} \leq \frac{17+\sqrt{33}}{2}<12$.

\section{Experimental results}

In this section, the denoising effect is evaluated by the signal to noise ratio (SNR), the peak signal to noise ratio (PSNR), the mean square error (MSE) and the structural similarity index (SSIM) [29]. They are defined as

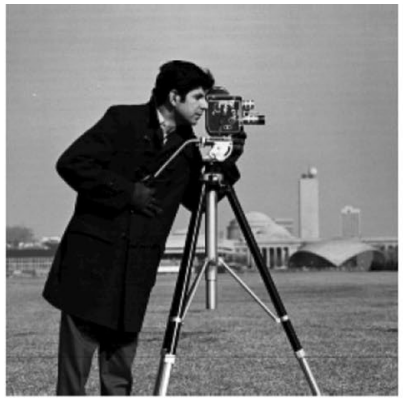

b

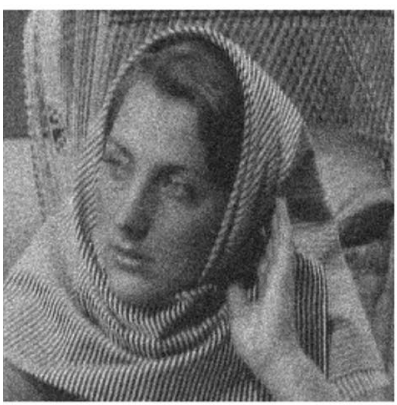

d

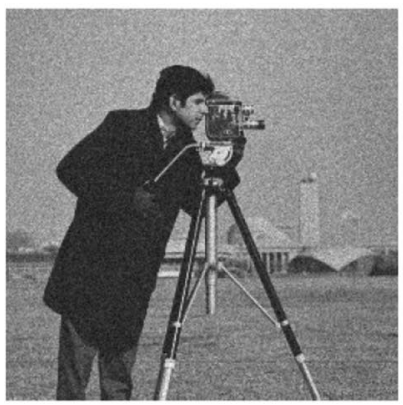

e

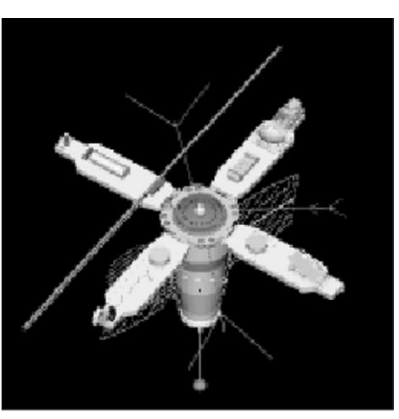

C

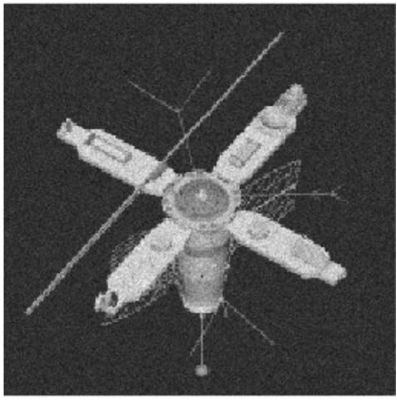

f 


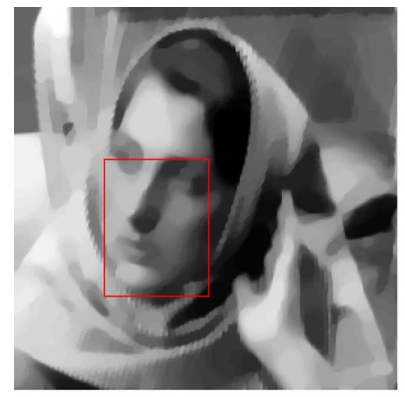

a

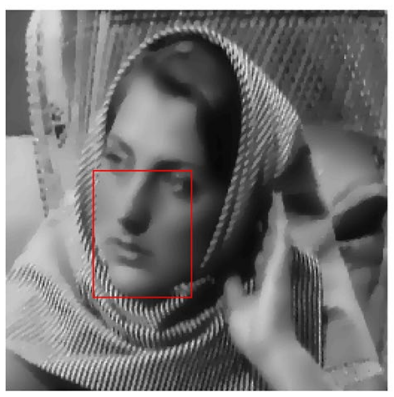

C

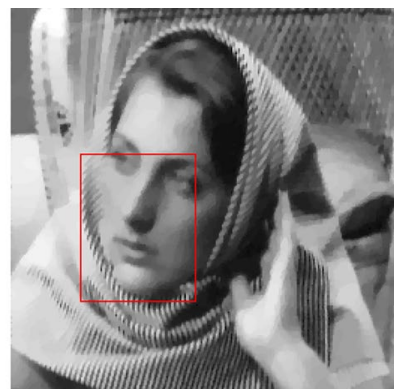

b

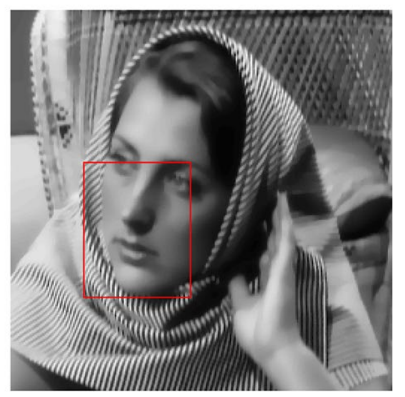

d
Fig. 2 The denoised Barbara images using four different methods. a TV-PD, b TV-AL, c TGV-Split, d our method

Table 1 The denoising effect comparison of Barbara image under Poisson noise

\begin{tabular}{lllll}
\hline Method & SNR & PSNR & MSE & SSIM \\
\hline TV-PD [30] & 15.71 & 21.94 & 415.54 & 0.3866 \\
TV-AL [31] & 18.94 & 25.17 & 197.52 & 0.6222 \\
TGV-Split [17] & 20.96 & 27.19 & 124.05 & 0.6536 \\
Our method & 25.73 & 31.97 & 41.35 & 0.7209 \\
\hline
\end{tabular}

$\mathrm{SNR}=20 \log _{10}\left(\frac{\|u\|_{2}}{\|\hat{u}-u\|_{2}}\right)$,

PSNR $=10 \log _{10}\left(\frac{\max _{1 \leq i \leq m, 1 \leq j \leq n}\left|u_{i, j}\right|^{2}}{\frac{1}{m n} \sum_{i=1}^{m} \sum_{j=1}^{n}\left(u_{i, j}-\hat{u}_{i, j}\right)^{2}}\right)$,

MSE $=\frac{1}{m n}\|u-\hat{u}\|_{2^{\prime}}^{2}$

$\operatorname{SSIM}=\frac{\left(2 \mu_{u} \mu_{\widehat{u}}+C_{1}\right)\left(2 \sigma_{u \hat{u}}+C_{2}\right)}{\left(\mu_{u}^{2}+\mu_{\widehat{u}}^{2}+C_{1}\right)\left(\sigma_{u}^{2}+\sigma_{\widehat{u}}^{2}+C_{2}\right)}$

where $u$ is the original clean image, $\hat{u}$ is the denoised image, the size of image is $m \times n, \mu_{u}$ and $\mu_{\widehat{u}}$ are averages of $u$ and $\hat{u}$, respectively. $\sigma_{u}$ and $\sigma_{\widehat{u}}$ are the variance of $u$ and $\widehat{u}$, respectively. $\sigma_{u \hat{u}}$ is the covariance of $u$ and $\hat{u} . C_{1}$ and $C_{2}$ are positive constants, we can assume that $C_{1}$ and $C_{2}$ are small enough to be ignored. Generally speaking, the larger

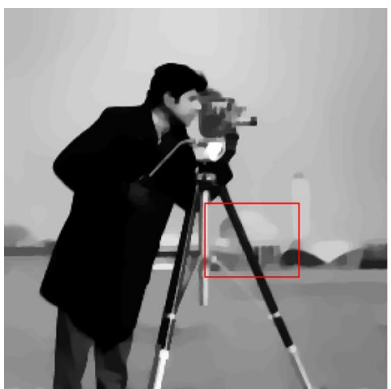

a

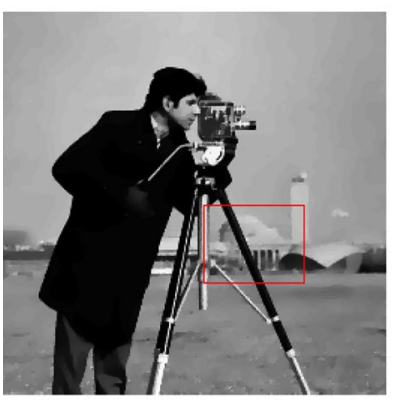

C

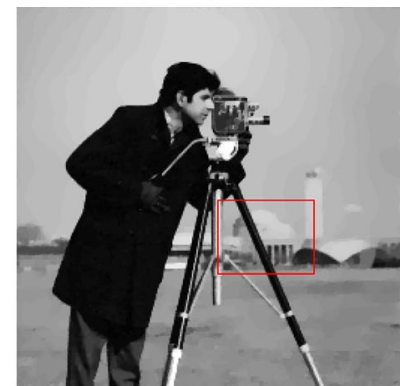

b

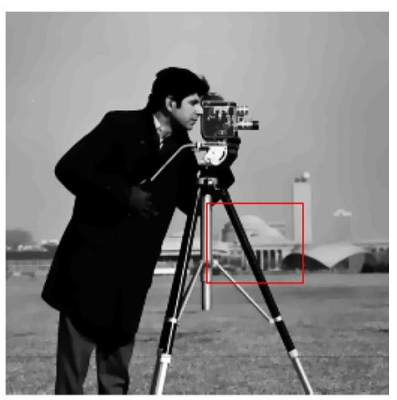

d
Fig. 3 The denoised cameraman images using four different methods. a TV-PD, b TV-AL, c TGV-Split, d our method

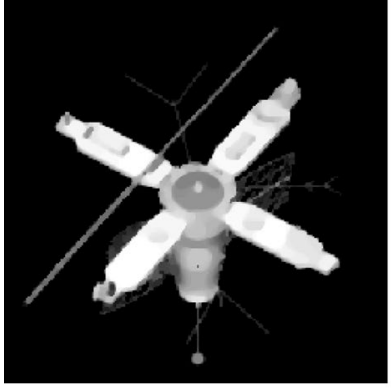

a

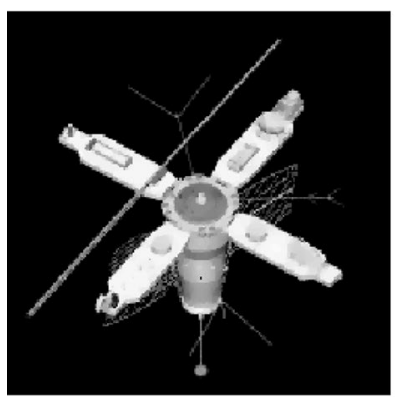

C

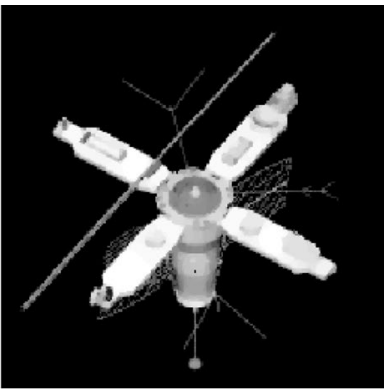

b

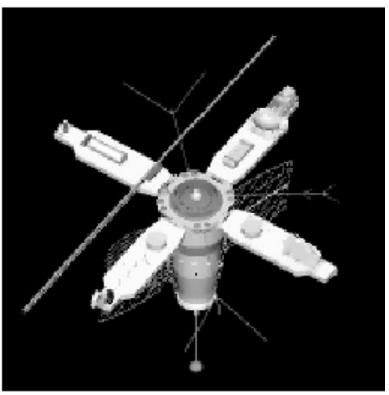

d
Fig. 4 The denoised satellite images using four different methods. a TV-PD, b TV-AL, c TGV-Split, d our method

the SNR, PSNR and SSIM, and the lower the MSE, the better the performance. 
Table 2 The denoising effect comparison of cameraman image under Poisson noise

\begin{tabular}{lllll}
\hline Method & SNR & PSNR & \multicolumn{1}{l}{ MSE } & \multicolumn{1}{l}{ SSIM } \\
\hline TV-PD [30] & 20.15 & 25.73 & 173.91 & 0.4694 \\
TV-AL [31] & 23.44 & 29.02 & 81.43 & 0.5479 \\
TGV-Split [17] & 24.98 & 30.56 & 57.17 & 0.5438 \\
Our method & 28.70 & 34.28 & 24.27 & 0.5843 \\
\hline
\end{tabular}

Table 3 The denoising effect comparison of satellite image under Poisson noise

\begin{tabular}{lllrl}
\hline Method & SNR & PSNR & \multicolumn{1}{l}{ MSE } & SSIM \\
\hline TV-PD [30] & 16.83 & 27.19 & 124.11 & 0.8931 \\
TV-AL [31] & 21.10 & 31.46 & 46.44 & 0.9435 \\
TGV-Split [17] & 24.20 & 34.56 & 22.74 & 0.9584 \\
Our method & 28.71 & 39.08 & 8.05 & 0.9620 \\
\hline
\end{tabular}

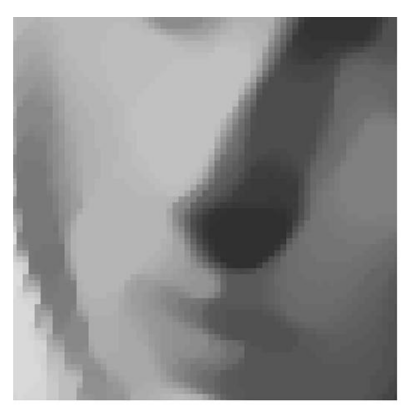

a

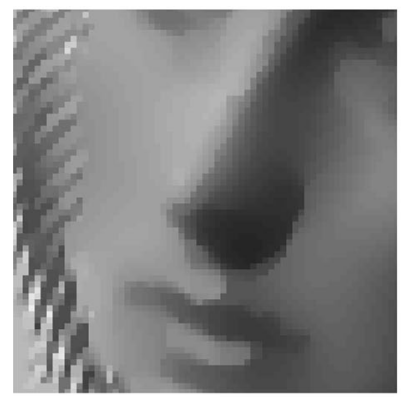

C

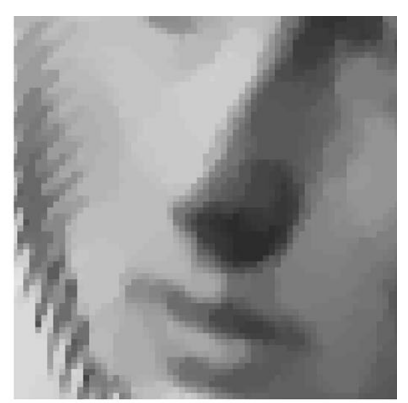

b

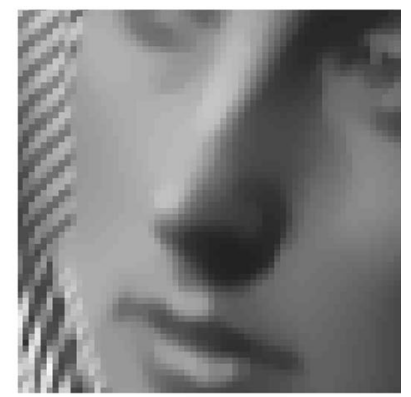

d
Fig. 5 Locally enlarged denoised Barbara images using four different methods. a TV-PD, b TV-AL, c TGV-Split, d our method

Here, our proposed method is compared with two existing TV-based methods and one TGV-based method. For simplicity, the method of [30] is called as TV-PD, the method of [31] is called as TV-AL, and the method of [17] is called as TGV-Split. Whether for the TV-based methods or the TGV-based method, we all use the stopping criterion

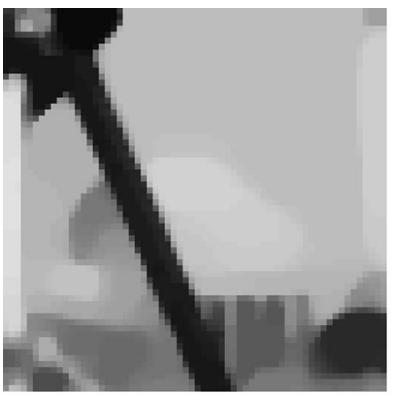

a

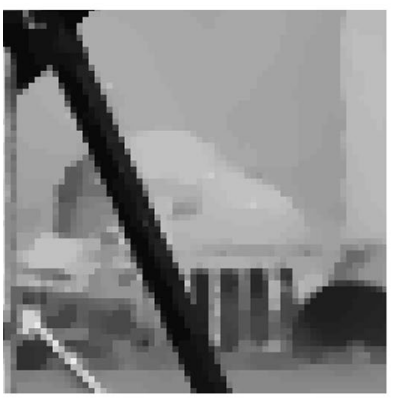

C

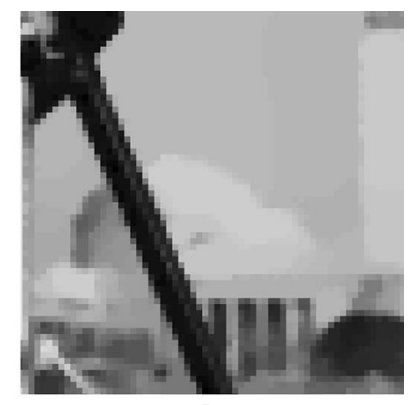

b

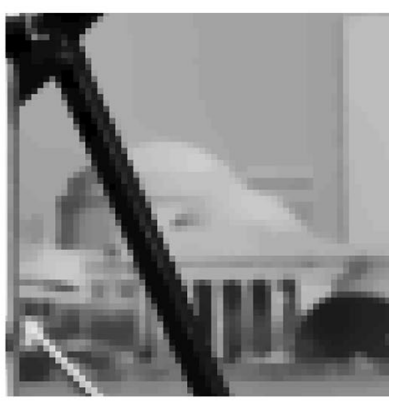

d
Fig. 6 Locally enlarged denoised cameraman images using four different methods. a TV-PD, b TV-AL, c TGV-Split, $\mathbf{d}$ our method

$\frac{\left\|u^{k+1}-u^{k}\right\|_{2}^{2}}{\left\|u^{k+1}\right\|_{2}^{2}} \leq 10^{-4}$,

or the iteration number larger than 200. The Barbara, cameraman and satellite images are used for testing, all with size $256 \times 256$. The poissrnd function in the statistics toolbox of MATLAB is used to generate the Poisson noise. All experiments were performed on Matlab R2014a, and Windows 10 with a $2.5 \mathrm{GHz}$ Intel Core i5 processor and $4 \mathrm{~GB}$ of RAM.

In Fig. 1, the original and noisy Barbara, cameraman and satellite images are displayed. The first row consists of the original Barbara, cameraman and satellite images, respectively. The second row are the noisy Barbara, cameraman and satellite images, respectively.

In Fig. 2, the denoised Barbara images obtained by four different methods are provided. Figure $2 a$ is implemented via the TV-PD method by setting the parameters $\lambda=1, \alpha_{1}=1, \alpha_{0}=2, s=1 / 3, t=1 / 4$, Fig. $2 \mathrm{~b}$ is implemented via the TV-AL method by setting the parameter $\lambda=0.15$, Fig. $2 c$ is the denoised Barbara image obtained by the TGV-Split method with the parameters $\lambda=10^{-5}, \alpha_{1}=1, \alpha_{0}=2$. Moreover, in Fig. $2 \mathrm{~d}$, the denoised Barbara image obtained by our proposed method with $\lambda=0.8, \alpha_{1}=1, \alpha_{0}=2, s=1 / 16, t=1, \sigma=2, c=0.02$ is provided. Here, as suggested in [4], the relation of two 
positive constants $\alpha_{0}$ and $\alpha_{1}$ is $\alpha_{0}=2 \alpha_{1}$. And the values of $\alpha_{1}$ and $\alpha_{0}$ are chosen as suggested in [4], which can guarantee to obtain better denoising effect. For the parameters $s$ and $t$, i.e., the step sizes of the primal and dual variables. We notice that only the TV-PD method and our method involve the step sizes $s$ and $t$. For the TV-PD method, as analyzed in [30], the step sizes should satisfy $s t<1 / 8$. In this paper, $s=1 / 3$ and $t=1 / 4$ are set for the TV-PD method. Not only can it satisfy $s t<1 / 8$, but also it can guarantee to obtain the better denoising effect. For the proposed method, according to the convergence analysis of Sect. 3.2, we know that $\|K\|_{2}^{2}<12$ and the step sizes should satisfy $s t\|K\|_{2}^{2}<1$, i.e., the step sizes $s$ and $t$ should satisfy $s t<1 / 12$. Here, the step sizes $s=1 / 16$ and $t=1$ are set for our proposed method. On the one hand, the default values of $s$ and $t$ for our proposed method satisfy the inequality $s t<1 / 12$. On the other hand, they can guarantee to obtain the better denoising effect. In general, there is no strict restriction on the parameters $c, \lambda$ and $\sigma$. But the choice of these parameters should be guaranteed to obtain better denoising effect. In this paper, the default values of $c, \lambda$ and $\sigma$ are obtained by many experiments, which can guarantee to obtain the better denoising effect. Among these parameters, the parameters $\alpha_{0}, \alpha_{1}, c$ and $\sigma$ are the most general parameters, and the parameters $\lambda$, $s$ and $t$ are the most critical parameters. If $\lambda$ is too large, the regularized solution is under-smoothed. But if $\lambda$ is too small, the regularized solution does not fit the given data properly. If default values of $s$ and $t$ are improper, the convergence of the TV-PD method and our proposed method can't be guaranteed, and the better denoising effect can't be obtained. Furthermore, the denoising effect comparison of Barbara image is displayed in Table 1. From Table 1, we can see that the better performance of our method in terms of possessing higher SNR, PSNR and SSIM values and lower MSE values than the TV-PD, TV-AL and TGV-Split methods.

Similarly, in Figs. 3 and 4, the denoised cameraman and satellite images obtained by four different methods are provided. Furthermore, in Tables 2 and 3, the denoising effect comparison of the cameraman and satellite images are provided, respectively. Compared with the denoised satellite images obtained by four different methods, see Fig. 4a-d, respectively. It is clear that our proposed method is effective in preserving the details of edges. From Tables 2 and 3 , whether for the cameraman or satellite image, our proposed method also has higher SNR, PSNR and SSIM values as well as lower MSE values over the TV-PD, TV-AL and TGVSplit methods. These also demonstrate the effectiveness of our proposed method.

Furthermore, the locally enlarged Barbara images using four different methods are provided in Fig. $5 a-d$, respectively. From Fig. 5a, b, it is clear to see that the denoised Barbara images obtained by the TV-PD and TV-AL methods cause the terrible staircase effect. Although the TGV-Split method has the ability of eliminating the staircase effect, see Fig. 5c, it causes the undesired edges blurring. However, from Fig. $5 \mathrm{~d}$, we can see that our scheme can eliminate the staircase effect and preserve the edges details. Meanwhile, in Fig. 6, the locally enlarged cameraman images obtained by four different methods are provided. From Figs. 3d and $6 \mathrm{~d}$, we can see that the denoised cameraman image obtained by our proposed WTGV model is more clear and natural than the TV-PD, TV-AL and TGV-Split methods.

The value of MSE which results from the proposed method is an order of magnitude lower than other methods demonstrates the outstanding denoising performance of our propsed method. From the definition of MSE, it is clear to see that the lower MSE value implies that the denoised image is closely to the original clean image. On the one hand, our proposed method can be seen as a TGV-based method, and the TV-PD and TV-AL methods can be seen as the TV-based methods. As is discussed in Sect. 1, we know that the TGV-based method can effectively eliminate the staircase effect which often occurs in the TV-based methods. Thus, it is reasonable to believe that our proposed method outperforms the TV-PD and TV-AL methods. On the other hand, a weight function is involved in our proposed method. For the weight function $g(x), c$ is a comparison factor which is used to determine the current pixel values belong to the smooth regions or the edges. For a default value $c$, the current pixel values belong to the edges when the value of $c\left|\nabla G_{\sigma} * f\right|^{2}$ is large, and this allows less regularization. Thus, the edges will be preserved better. In contrast, the current pixel values belong to the smooth regions when the value of $c\left|\nabla G_{\sigma} * f\right|^{2}$ is small, and this allows more regularization. Thus, the noise will be efficiently removed. That is to say, the weight function $g(x)$ can balance the noise removal and edges preservation. Furthermore, from Figs. $5 c$ and $6 c$, it is clear to see that although the TGV-Split method can effectively eliminate the staircase effect, it may produces over-smoothing edges. As is discussed above, it is clear that our proposed WTGV model can effectively preserve the details of edges. Thus, we have reason to believe that the proposed model outperforms the TGV-Split method. Based on what is mentioned above, it is no wonder that in Tables 1,2 and 3, the values of MSE which result from the proposed method are an order of magnitude lower than the TV-PD, TV-AL and TGV-Split methods.

\section{Conclusion}

In this paper, we proposed a novel WTGV model for Poisson noise removal. By introducing two dual variables, we reformulate the WTGV norm as a dual term. Then, the 
WTGV minimization problem is transformed into a minimax problem by using the dual term of the WTGV norm. Meanwhile, the high efficient Chambolle-Pock's first-order primal-dual algorithm is used to compute the saddle point of the minimax problem. Experimental results show that our proposed model is effective in edges preservation and staircase effect elimination. For the WTGV model, the regularization parameter selection is a difficult problem which needs further research.

\section{Compliance with ethical standards}

Conflict of interest The authors declare that they have no conflict of interest, whether financial or non-financial.

Human and animal rights This research did not involve human participants and animals.

\section{References}

1. Rudin L, Osher S, Fatemi E (1992) Nonlinear total variation based noise removal algorithms. Phys D 60(1-4):259-268

2. Strong DM, Blomgren P, Chan TF (1997) Spatially adaptive local feature-driven total variation minimizing image restoration. Proc SPIE 3167:222-233

3. Strong M, Moroni D (1997) Adaptive total variation minimizing image restoration. UCLA CAM report

4. Bredies K, Kunisch K, Pock TT (2010) Total generalized variation. SIAM J Imaging Sci 3(3):492-526

5. Florian K, Kristian B, Thomas P et al (2011) Second order total generalized variation (TGV) for MRI. Magn Reson Med 65(2):480-491

6. Bratsolis E, Sigelle M (2001) A spatial regularization method preserving local photometry for Richardson-Lucy restoration. Astron Astrophys 375(3):1120-1128

7. Starck JL, Murtagh F (2007) Astronomical image and data analysis. Springer, Berlin

8. Dey N, Blanc-Feraud L, Zimmer C et al (2010) Richardson-Lucy algorithm with total variation regularization for 3D confocal microscope deconvolution. Microsc Res Tech 69(4):260-266

9. Kryvanos A, Hesser J, Steidl G (2005) Nonlinear image restoration methods for marker extraction in 3D fluorescent microscopy. Proc SPIE 5674:432-443

10. Sarder P, Nehorai A (2006) Deconvolution methods for 3D fluorescence microscopy images. IEEE Signal Proc Mag 23(3):32-45

11. Rodrigues I, Sanches J, Bioucas-Dias J (2008) Denoising of medical images corrupted by Poisson noise. In: IEEE international conference on image processing

12. Trinh $H$, Luong $M$, Dibos $F$ et al (2015) An effective examplebased learning method for denoising of medical images corrupted by heavy Gaussian noise and Poisson noise. In: IEEE international conference on image processing
13. Le T, Chartrand R, Asaki TJ (2007) A variational approach to reconstructing images corrupted by Poisson noise. J Math Imaging Vis 27(3):257-263

14. Sawatzky A, Brune C, Kosters T et al (2013) EM-TV methods for inverse problems with Poisson noise. Springer, Cham

15. Setzer S, Steidl G, Teuber T (2010) Deblurring poissonian images by split bregman techniques. J Vis Commun Image R 21(3):193-199

16. Bonettini S, Benfenati A, Ruggiero V (2014) Primal-dual first order methods for total variation image restoration in presence of Poisson noise. In: IEEE international conference on image processing

17. Li HB, Wang JY, Dou HX (2016) Second-order TGV model for Poisson noise image restoration. Springerplus 5(1):1272

18. Chen Y, Wunderli T (2002) Adaptive total variation for image restoration in BV space. J Math Anal Appl 272(1):117-137

19. Bredies K (2014) Recovering piecewise smooth multichannel images by minimization of convex functionals with total generalized variation penalty. In: Bruhn A, Pock T, Tai XC (eds) Efficient algorithms for global optimization methods in computer vision. Lecture notes in computer science, vol 8293. Springer, Berlin

20. Bertsekas D (2009) Convex optimization theory. Athena Scientific, Belmont

21. Chen G, Teboulle M (1994) A proximal-based decomposition method for convex minimization problems. Math Program 64(1-3):81-101

22. Combettes PL, Pesquet JC (2008) A proximal decomposition method for solving convex variational inverse problems. Inverse Probl 24(6):065014

23. Eckstein J, Bertsekas DP (1992) On the Douglas-Rachford splitting method and the proximal point algorithm for maximal monotone operators. Math Program 55(1-3):293-318

24. Franois-Xavier D, Jalal MF, Jean-Luc S (2009) A proximal iteration for deconvolving Poisson noisy images using sparse representations. IEEE Trans Image Process 18(2):310-321

25. Rockafellar RT (1976) Augmented Lagrangians and applications of the proximal point algorithm in convex programming. Math Oper Res 1(2):97-116

26. Zhu M, Chan TF (2008) An efficient primal-dual hybrid gradient algorithm for total variation image restoration. UCLA CAM report

27. Chambolle A, PockT (2011) A first-order primal-dual algorithm for convex problems with applications to imaging. J Math Imaging Vis 40(1):120-145

28. Chan R, Chan T, Yip A (2011) Numerical methods and applications in total variation image restoration. Handbook of mathematical methods in imaging

29. Wang Z, Bovik AC, Sheikh HR et al (2004) Image quality assessment: from error visibility to structural similarity. IEEE Trans Image Process 13(4):600-612

30. Wen YW, Chan RH, Zeng TY (2015) Primal-dual algorithms for total variation based image restoration under Poisson noise. Sci China Math 59(1):141-160

31. Jiang L, Huang J, Lv XG et al (2015) Alternating direction method for the high-order total variation-based Poisson noise removal problem. Numer Algor 69(3):495-516

Publisher's Note Springer Nature remains neutral with regard to jurisdictional claims in published maps and institutional affiliations. 